\title{
Blind channel deconvolution of real world signals using source separation techniques
}

\author{
Jordi Solé-Casals $^{1}$, Enric Monte-Moreno ${ }^{2}$ \\ ${ }^{1}$ Signal Processing Group, University of Vic, Sagrada Família 7, 08500, Vic (Catalonia) \\ http://www.uvic.es/eps/recerca/processament/ca/inici.html \\ ${ }^{2}$ Polytechnic University of Catalonia, Campus Nord, UPC, Barcelona (Catalonia) \\ enricegps.tsc.upc.es \\ http://gps-tsc.upc.es/veu/personal/enric/enric.html
}

\begin{abstract}
In this paper we present a method for blind deconvolution of linear channels based on source separation techniques, for real word signals. This technique applied to blind deconvolution problems is based in exploiting not the spatial independence between signals but the temporal independence between samples of the signal. Our objective is to minimize the mutual information between samples of the output in order to retrieve the original signal. In order to make use of use this idea the input signal must be a non-Gaussian i.i.d. signal. Because most real world signals do not have this i.i.d. nature, we will need to preprocess the original signal before the transmission into the channel. Likewise we should assure that the transmitted signal has non-Gaussian statistics in order to achieve the correct function of the algorithm. The strategy used for this preprocessing will be presented in this paper. If the receiver has the inverse of the preprocess, the original signal can be reconstructed without the convolutive distortion.
\end{abstract}

\section{Introduction}

Many researches have been done in the identification and/or the inversion of linear and nonlinear systems. These works assume that both the input and the output of the system are available [14]; they are based on higher-order input/output crosscorrelation [3], bispectrum estimation [12,13] or on the application of the Bussgang and Prices theorems [4, 9] for nonlinear systems with Gaussian inputs. However, in real world situations, one often does not have access to the distortion input. In this case, blind identification of the system becomes the only way to solve the problem. In this paper we propose to adapt the method presented in $[17,18]$ for blind deconvolution by means of source separation techniques, to the case of real world signals which are non i.i.d. This is done by means of a stage of preprocessing before sending the signal and post-processing stage after the reception and deconvolution. The paper is organized as follows. The source separation problem is described in Section 2. The model and assumptions for applying these techniques to blind deconvolution are presented in Section 3. Section 4 contains the proposed preprocessing and post- 
processing stages, and the simulation results are presented in Section 5, before concluding in Section 6.

\section{Source Separation review}

The problem of source separation may be formulated as the recovering of a set of unknown independent signals from the observations of mixtures of them without any prior information about either the sources or the mixture $[10,6]$. The strategy used in this kind of problems is based in obtaining signals as independent as possible at the output of the system. In the bibliography multiple algorithms have been proposed for solving the problem of source separation in instantaneous linear mixtures. These algorithms range from neural networks based methods [1], cumulants or moments methods [7, 5], geometric methods [15] or information theoretic methods [2]. In real world situations, however, the majority of mixtures can not be modeled as instantaneous and/or linear. This is the case of the convolutive mixtures, where the effect of channel from source to sensor is modeled by a filter [11]. Also the case of the postnonlinear (PNL) mixtures, where the sensor is modeled as a system that performs a linear mixture of sources plus a nonlinear function applied to its output, in order to take into account the possible non-linearity of the sensor (saturation, etc.) [16].

Mathematically, we can write the observed signals in source separation problem of instantaneous and linear mixtures as (see figure 1):

$$
e_{i}(t)=\sum_{j=1}^{n} a_{i j} s_{j}(t)
$$

where $\mathbf{A}=\left\{a_{i j}\right\}$ is the mixing matrix. It is well known that such a system is blindly invertible if the source signals are statistically independent and we have no more than one Gaussian signal.

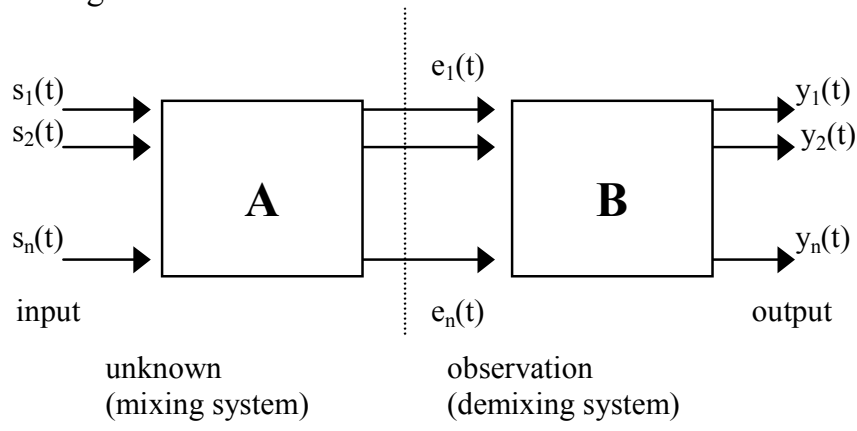

Fig. 1. Block diagram of the mixture system and blind demixing system. Both matrix A and signals $\mathrm{s}_{\mathrm{i}}(\mathrm{t})$ on the mixture process are unknown.

A solution may be found by minimizing the mutual information function between the outputs of the system $y_{\mathrm{i}}$ : 


$$
\min _{B}[I(y)]=\min _{B}\left[\sum_{i=1}^{n} H\left(y_{i}\right)-H(e)-\ln |\operatorname{det}(B)|\right]
$$

A related problem with blind separation is the case of blind deconvolution, which is presented in figure 2, and can be expressed in the framework of Equation (1). Development of this framework is presented in the following section.

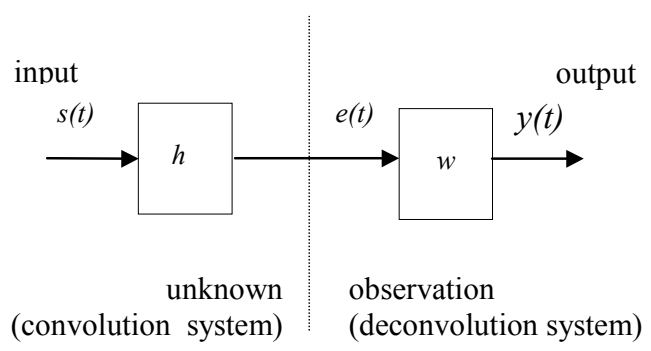

Fig. 2. Block diagram of the convolution system and blind deconvolution system. Both filter $h$ and signal $s(\mathrm{t})$ on the convolution process are unknown.

\section{Model and assumptions}

We suppose that the input of the system $S=\{s(t)\}$ is an unknown non-Gaussian independent and identically distributed (i.i.d.) process. We are concerned by the estimation of $s(t)$ from the system's output $e$. This implies the blind inversion of a filter. From figure 2, we can write the output of filter $h$ in a similar form that obtained in source separation problem, Equation 1, but now with vectors and matrix of infinite dimension:

$$
e=H s
$$

where:

$$
H=\left[\begin{array}{ccccc}
\cdots & \ldots & \ldots & \ldots & \ldots \\
\cdots & h(t+1) & h(t) & h(t-1) & \ldots \\
\ldots & h(t+2) & h(t+1) & h(t) & \ldots \\
\cdots & \ldots & \ldots & \ldots & \ldots
\end{array}\right]
$$

is a Toeplitz matrix of infinite dimension and represents the action of filter $h$ to the signal $s(t)$. This matrix $h$ is nonsingular provided that the filter $h$ is invertible, i.e. $h^{-1}$ exists and satisfies $h^{-1} * h=h * h^{-1}=\delta_{0}$, where $\delta_{0}$ is de Dirac impulse. The solution to invert this systems and the more general nonlinear systems (Wiener systems) are presented and studied in $[17,18]$ where a Quasi-nonparametric method is presented. 
In the particular case of windowed signals, $h$ is of finite length, and the product $h^{-1} * h$ yields a Dirac impulse with a delay.

\subsection{Summary of the deconvolution algorithm}

From figure 2 we can write the mutual information of the output of the filter $w$ using the notion of entropy rates of stochastic processes [12] as:

$$
\begin{aligned}
I(Y) & =\lim _{T \rightarrow \infty} \frac{1}{2 T+1}\left\{\sum_{t=-T}^{T} H(y(t))-H\left(y_{-T}, \ldots, y_{T}\right)\right\} \\
& =H(y(\tau))-H(Y)
\end{aligned}
$$

where $\tau$ is arbitrary due to the stationary assumption. The input signal $S=\{s(t)\}$ is an unknown non-Gaussian i.i.d. process, $Y=\{y(t)\}$ is the output process and $\mathbf{y}$ denotes a vector of infinite dimension whose $\mathrm{t}$-th entry is $y(t)$. We shall notice that $I(Y)$ is always positive and vanishes when $Y$ is i.i.d.

After some algebra, Equation (2) can be rewritten as [10]:

$$
I(Y)=H(y(\tau))-\frac{1}{2 \pi} \int_{0}^{2 \pi} \log \left|\sum_{t=-\infty}^{+\infty} w(t) e^{-j t \theta}\right| d \theta-E[\mathcal{E}]
$$

To derive the optimization algorithm we need the derivative of $\mathrm{I}(Y)$ with respect to the coefficients of $w$ filter. For the first term of Equation (3) we have:

$$
\frac{\partial H(y(\tau))}{\partial w(t)}=-E\left[\frac{\partial y(\tau)}{\partial w(t)} \psi_{y}(y(\tau))\right]=-E\left[e(\tau-t) \psi_{y}(y(\tau))\right]
$$

where $\psi_{y}(u)=\left(\log P_{y}\right)^{\prime}(u)$ is the score function. The second term is:

$$
\frac{\partial}{\partial w(t)} \frac{1}{2 \pi} \int_{0}^{2 \pi} \log \left|\sum_{n=-\infty}^{+\infty} w(n) e^{-j n \theta}\right| d \theta=\frac{1}{2 \pi} \int_{0}^{2 \pi} \frac{e^{-j t \theta}}{\sum_{n=-\infty}^{+\infty} w(n) e^{-j n \theta}} d \theta
$$

where one recognizes the $\{-\mathrm{t}\}$-th coefficient of the inverse of the filter $w$, which we denote $\bar{w}(-t)$. Combining Equations (5) and (6) leads to:

$$
\frac{\partial I(Y)}{\partial w(t)}=-E\left[x(\tau-t) \psi_{y}(y(\tau))\right]-\bar{w}(-t)
$$

that is the gradient of $I(Y)$ with respect to $w(t)$. Using the concept of natural or relative gradient, the gradient descendent algorithm will be finally as:

$$
w \leftarrow w+\mu\left\{E\left[x(\tau-t) \psi_{y}(y(\tau))\right]+\delta\right\} * w
$$

It is important to notice that blind deconvolution is a different problem than source separation. In our blind deconvolution scenario the objective is to recover an un- 
known signal filtered by an unknown filter, using only the received (observed) signal. The main idea, proposed in $[17,18]$ is to use independence criteria, as in source separation problems, for deal with this problem. The relationship between source separation and deconvolution is shown in figure 3.

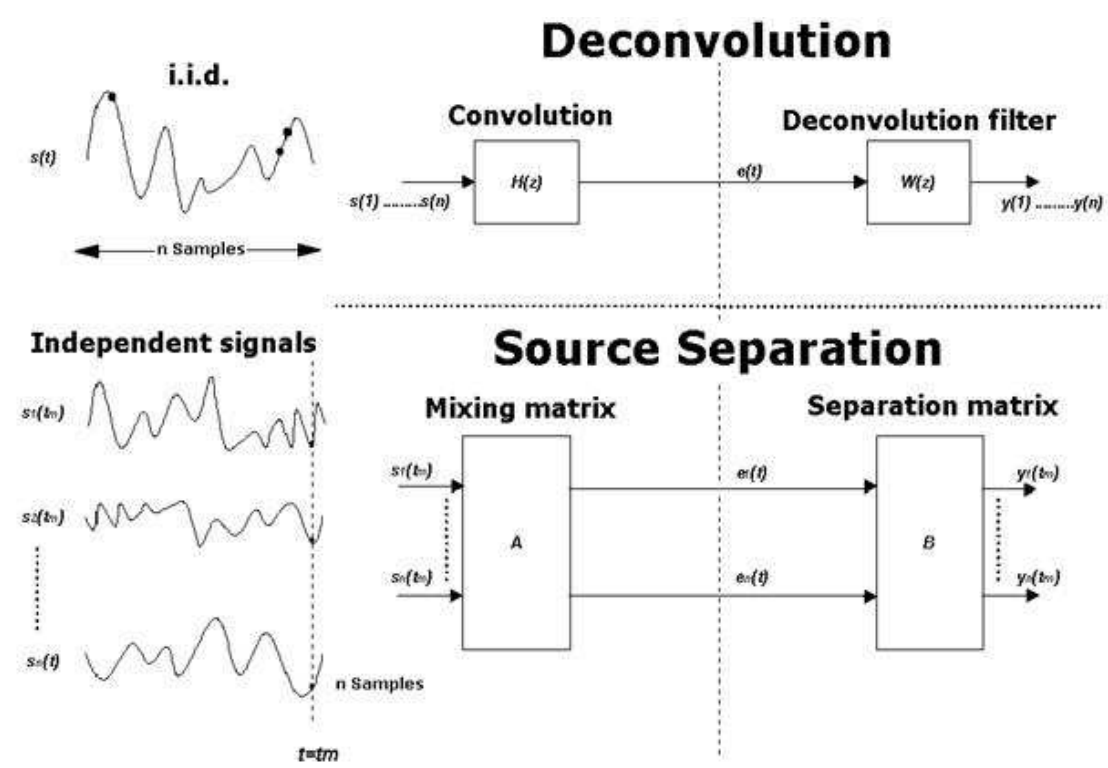

Fig. 3. Relationship between deconvolution and source separation. In source separation we force spatial independence between samples of different signals (at the same time instant). On the other hand, in deconvolution we impose this independence between samples of the unique signal at different times (time independence).

\section{Application to real world signals}

When signals are sent from the transmitter to the receiver, they suffer changes in their waveform form due to the effect of the transmission channel. In real systems, usually the transmission channel is unknown, and therefore the effect over the signals is uncertain. In this case, the use of an equalizer is necessary in order to ensure that the received signal is exactly the sent signal. Our proposed method uses directly the signal of interest to blindly estimate the inverse of the transmission channel, without the need of any other technique for equalizing the channel. In order to apply this method to real world signals we have to deal, as shown in previous Section with the fact that the signals are usually not i.i.d., so we can not use this method directly. In order to use it, we need to preprocess the input signal to ensure its temporal independence 
between samples and also to ensure its non-Gaussian distribution. In this section we present these preprocessing and post-processing stages in experiments done with speech signals.

\subsection{Whitening of the signal}

Speech signals have a clear correlation structure, and therefore are not i.i.d. In order to use source separation techniques for blind deconvolution a preprocessing stage is necessary and it consists on a whitening of the original signal by means of an inverse LPC filter.

The use of a LPC preprocessing is useful in the case of signals that can be modeled as autoregressive processes. This is the case of speech signal or string and wind instruments, which were our test signals. In figure 4 we can see the autocorrelation sequence of a speech segment corresponding to the vowel /a/, before and after the whitening filter. Note that the prediction residual has peaks at multiples of the pitch frequency, and the residual in between these peaks is reduced.
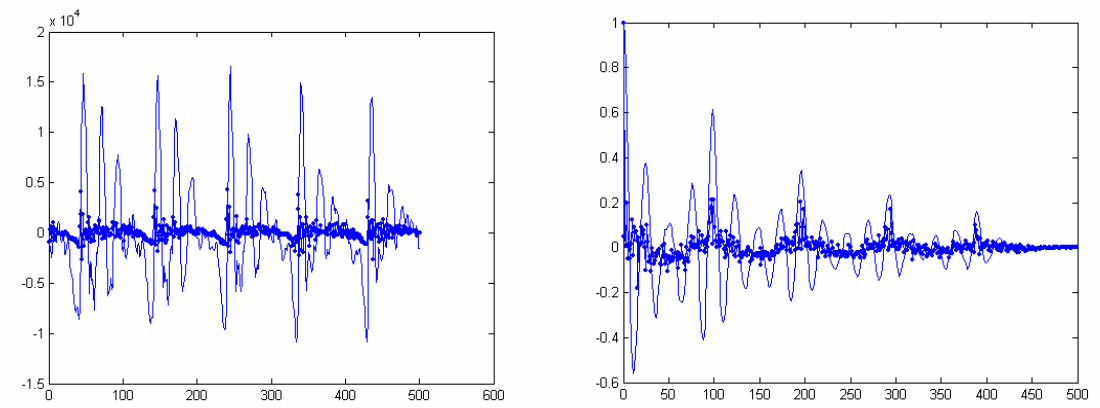

Fig. 4. Left: wave form of Vowel /a/ and the prediction residual. Right: Comparison of the normalized autocorrelations of the vowel signal and the residual of the LPC.

The resulting residual after the whitening filter is an i.i.d. signal with a Gaussian distribution. Consequently it will be necessary to change de probability density function of the i.i.d. signal in order to acquire the necessary conditions to apply the deconvolution process on the receiver.

\subsection{Des-Gaussianity of the signal}

In figure 5 we show the quantiles of the signal and the residual vs. the quantiles of a Gaussian distribution. One can see that the whitened signal has a nearly linear relation and is symmetric. For the purposes of the algorithm that we propose the signal can be considered Gaussian.

In order to change its probability density function, we propose a method based on the following observations: 
1. Speech signals are periodic signals, with a fundamental period (pitch frequency).

2. A whitening filter removes all the components and keeps only the nonpredictable part that corresponds precisely to the excitation of the speech signal (the pitch), and to the excitation of the instrument, with its fundamental period.

Starting from these observations we propose the following method:

1. Normalize the whitened signal provided by a LPC filter in a way that the maximums associated whit the periodic excitation been around \pm 1 .

2. Pass the result signal trough a nonlinear function that maintains the peak values but modify substantially all the other values, therefore the pdf. We propose two functions for this task:

2.1. exponential function $x(\cdot)^{n}$ : attenuate all the values between two consecutive peaks.

2.2. $\tanh (\cdot)$ : amplify all the values between two consecutive peaks.

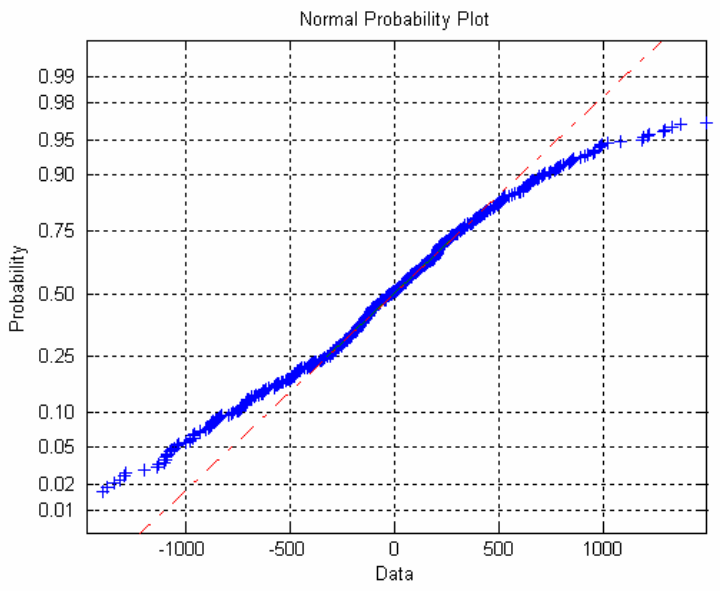

Fig. 5. Plot of the quantiles of the whitened signal versus probability of a Gaussian. One can observe the Gaussianity of the distribution.

The effect of this process will be that the output signal will maintain important parts of the signal (the peaks of the series) and will change the form of the distribution. In the next figure we can see the proposed method: 


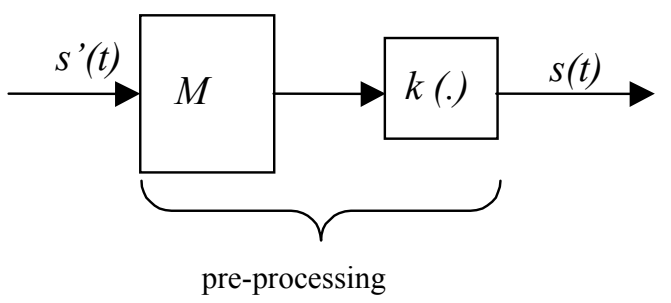

Fig. 6. The proposed preprocessing stage, composed by a whitening filter $M$ and a nonlinear function $k(\cdot)$ preserving the most important part of the input signal. This stage is applied before sending the (unknown) signal through the transmitter channel.

\section{Experimental results}

The input signal is a fragment of speech signal, preprocessed as shown in the diagram of figure 5. This is the signal that we want to transmit through an unknown channel. Our objective is to recover the original signal $s^{\prime}(t)$ only from the received observation $e(t)$.

Consider, now, the filter $h$ as a FIR filter with coefficients: $h=[0.826$,$0.165,0.851,0.165,0.810]$. Figure 8 show that $h$ has two zeros outside the unit circle, which indicates that $h$ is non-minimum phase. The system that we propose will always yield a stable equalizer because it computes a FIR approximation of the IIR optimal solution. The algorithm was provided with a signal of size $T=500$. The size of the impulse response of $w$ was set to 81. In the pre-processing stage the length of LPC was fixed at 12 coefficients and nonlinear function (des-Gaussianity) was $k(u)=$ $u^{3}$.

In figure 9, on the left we show the prediction residual, before and after a cubic non-linearity. On the right we show a quantile plot of the residual vs. a Gaussian distribution. It can be seen that it does not follow a linear relation. The results showed in figure 10 prove the good behavior of the proposed algorithm, i.e. we perform correctly the blind inversion of the filter (channel). The recovered signal at the output of the system has the spectrum showed in figure 11. We can see how, although we have modified in a nonlinear manner the input signal in the preprocess stage, the spectrum matches the original because non-linear function $k(\cdot)$ preserve the structure of the residual (non-predictable part) of the signal. The difference between harmonic peaks of speech signal and background noise is about $40 \mathrm{~dB}$. 


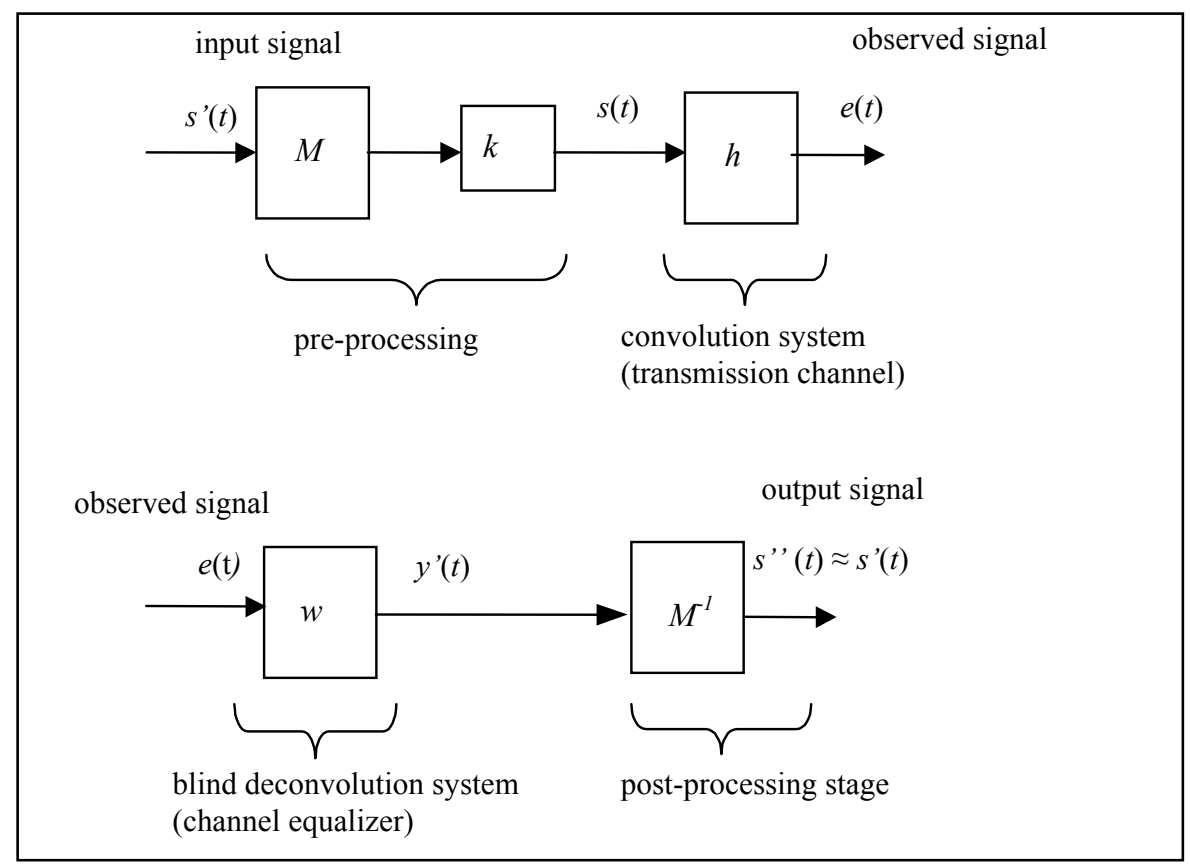

Fig. 7. On the upper part of the figure, the proposed preprocessing stage, and the convolution system. On the lower part, the blind deconvolution system and the post-processing stage.

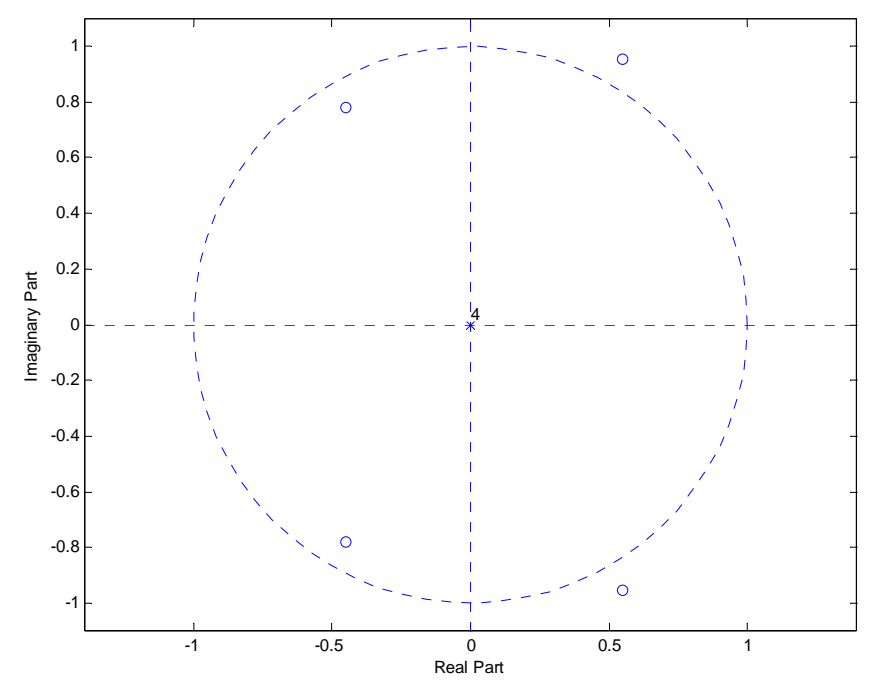

Fig. 8. Poles and zeros of h. We observe two zeros outside the unit circle, so the filter is of non-minimum phase. 

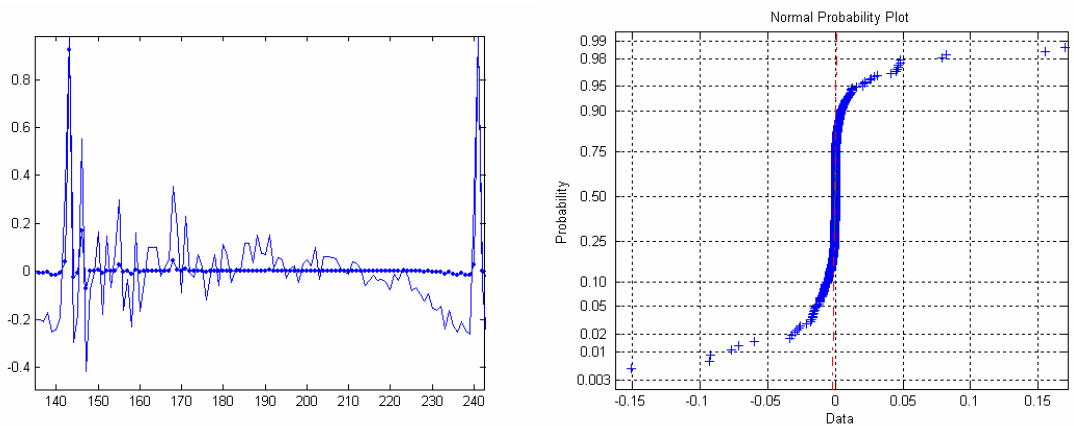

Fig. 9. On the left, the LPC residue of the signal superposed with the residual after the nonlinear operation. On the right, the quantile of the processed residual versus the quantiles of a Gaussian distribution.

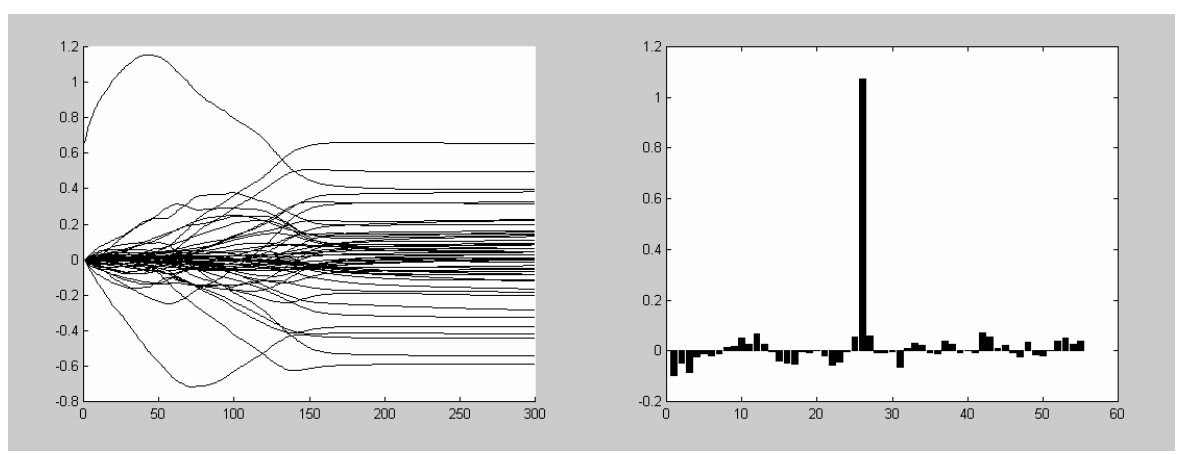

Fig. 10. On the left, filter coefficients evolution of the inverse filter $w$. The convergence is attained at 150 iterations approx. On the right, convolution between filter $h$ and its estimated inverse $w$. The result is clearly a delta function.

Similar experiments have been done with music signals, which are also non i.i.d. signals and have strong correlations between samples due to the resonance characteristics of the instruments. In this case, similarly to the previous experiments, the results are quite good, using the same parameters (non-linear function $k$, LPC order, etc.).

\section{Summary}

In this paper we have presented a method for blind deconvolution of channel applied to real world signals. This method is based on source separation techniques, which can estimate the inverse of a channel if the original signal is non-Gaussian and i.i.d. In order to apply this result to real world signals we will need to preprocess the original signal using a whitening stage by means of an inverse LPC filter and applying 
after a non-linear function for des-Gaussianity the signal. Thereby we modify the density probability function without changing the non predictable part of the signal. In reception, after the deconvolution of the signal, we need a post-processing stage by means of the inverse of the LPC filter in order to reconstruct the original signal.

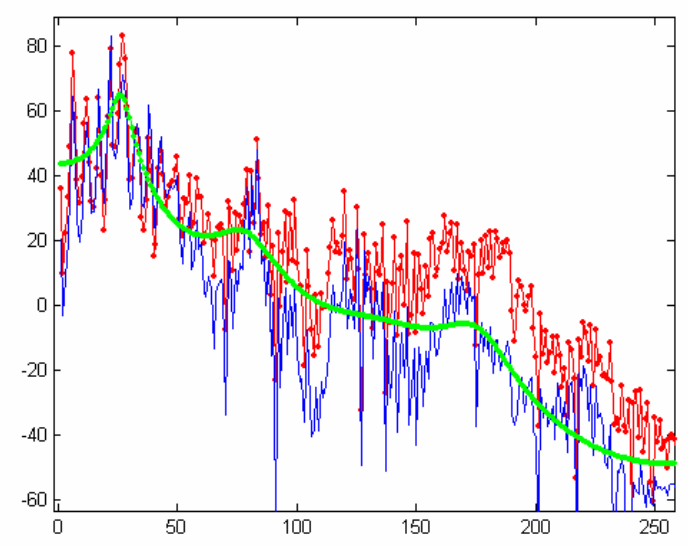

Fig. 11. Input signal spectrum (continuous line), output signal spectrum (dotted line) and LPC spectrum (Thick line). We can observe the similarity of these spectrums at low frequencies. The main difference is related to the high part of the spectrum of the reconstructed signal, which is due to the fact that the LPC reconstruction allocates resources to the parts of the spectrum with high energies

For a future works we are studying other possibilities in order to apply source separation techniques to linear or non-linear channel deconvolution problems with real world signals. Our preliminary work indicated that we can effectively invert the nonlinear convolution by means of source separation techniques $[10,11]$ but for a non stationary signal it is necessary to study how we can preprocess this signals to insure the i.i.d. and non Gaussian distribution necessary conditions to apply these techniques.

\section{Acknowledgment}

This work has been in part supported by the University of Vic under de grant R0912 and by the Spanish CICyT project ALIADO. 


\section{References}

1. Amari, S., Cichocki A., Yang, H.H., "A new learning algorithm for blind signal separation", NIPS 95, MIT Press, 8, 1996

2. Bell, A.J., Sejnowski T.J., "An information-maximization approach to blind separation and blind deconvolution", Neural Computation 7, 1995.

3. S.A. Bellings, S.Y. Fakhouri. Identification of a class of nonlinear systems using correlation analysis. Proc. IEEE, 66 pp. 691-697 (1978)

4. E.D. Boer. Cross-correlation function of a bandpass nonlinear network. Proc. IEEE, 64 pp. 1443-1444 (1976)

5. Cardoso, J.P., "Source separation using higher order moments", in Proc. ICASSP, 1989.

6. Comon, P., “Independent component analysis, a new concept?", Signal Processing 36, 1994.

7. Comon, P., "Separation of sources using higher order cumulants" Advanced Algorithms and Architectures for Signal Processing, 1989.

8. Cover, T.M., Thomas, J.A., Elements of Information Theory. Wiley Series in Telecommunications, 1991.

9. G. Jacoviti, A. Neri, R. Cusani. Methods for estimating the autocorrelation function of complex stationary process. IEEE Trans. ASSP, 35, pp. 1126-1138 (1987)

10. Jutten, C., Hérault, J. ,"Blind separation of sources, Part I: An adaptive algorithm based on neuromimetic architecture", Signal Processing 24, 1991.

11. Nguyen Thi, H.L., Jutten, C., "Blind source separation for convolutive mixtures", IEEE Transactions on Signal Processing, vol. 45, 2, 1995.

12. C.L. Nikias, A.P. Petropulu. Higher-Order Spectra Analysis - A Nonlinear Signal processing Framework. Englewood Cliffs, NJ: Prentice-Hall (1993)

13. C.L. Nikias, M.R.Raghuveer. Bispectrum estimation: A digital signal processing framework. Proc. IEEE, 75 pp. 869-890 (1987)

14. S. Prakriya, D. Hatzinakos. Blind identification of LTI-ZMNL-LTI nonlinear channel models. Biol. Cybern., 55 pp. 135-144 (1985)

15. Puntonet, C., Mansour A., Jutten C., "A geometrical algorithm for blind separation of sources", GRETSI, 1995

16. Taleb, A., Jutten, C., "Nonlinear source separation: the post-nonlinear mixtures", in Proc. ESANN, 1997

17. Taleb, A., Solé, J., Jutten, C., "Blind Inversion of Wiener Systems", in Proc. IWANN, 1999

18. Taleb, A., Solé, J., Jutten, C., "Quasi-Nonparametric Blind Inversion of Wiener Systems”, IEEE Transactions on Signal Processing, 49, n5, pp.917-924, 2001 